\title{
REGULARIZATION FOR LOZANOVSKIĬ'S TYPE FACTORIZATION WITH APPLICATIONS
}

\author{
Karol Leśnik, Lech Maligranda and Paweł Mleczko
}

Poznań University of Technology, Institute of Mathematics

ul. Piotrowo 3a, 60-965 Poznań, Poland; klesnik@vp.pl

Luleå University of Technology, Department of Engineering Sciences and Mathematics SE-971 87 Luleå, Sweden; lech.maligranda@ltu.se

Adam Mickiewicz University in Poznań, Faculty of Mathematics and Computer Science ul. Uniwersytetu Poznańskiego 4, 61-614 Poznań, Poland; pml@amu.edu.pl

\begin{abstract}
We say that a function space $Z$ is factorable by $X$ when there exists a third function space $Y$ such that each $f$ from $Z$ admits factorization $f=g h$, where $g, h$ belong to $X, Y$, respectively, and $\|f\|_{Z} \approx\|g\|_{X}\|h\|_{Y}$. We consider a problem of regularization of such a factorization; namely, suppose that $f$ like above satisfies some additional regularity condition (i.e., is holomorphic, smooth or is a simple function). May $g, h$ be chosen to have the same property? Answer to such a question when $f$ is holomorphic leads us to factorization of Hardy type spaces. We also apply these considerations to get factorization for Toeplitz operators on Hardy spaces.
\end{abstract}

\section{Introduction}

It is evident that each function in $L^{1}$ may be written as a pointwise product of two functions, one from $L^{p}$, the second from $L^{p^{\prime}}$, where, as usual, $p^{\prime}$ is conjugate of $p$. Moreover, factors may be chosen in such a way that the norm of $L^{1}$ function is equal to the product of norms of factors. This statement may be written as $L^{1} \equiv L^{p} \odot L^{p^{\prime}}$. The celebrated Lozanovskiu factorization theorem states that for an arbitrary Banach function lattice $X$, there holds $L^{1} \equiv X \odot X^{\prime}$, where $X^{\prime}$ is the Köthe dual of $X$ (see [Lo69, Theorem 6]; cf. also [Ma89, p. 185]). An extension of this idea leads to the question when given two (quasi-)Banach function lattices $Z, X$, there is a third one, say $Y$, such that $Z=X \odot Y$ ? In contrary to the case of $Z=L^{1}$, such a space may not exist. Nevertheless, when it does exist, it has to be equal (up to the Fatou property and equivalent norms) to the space of pointwise multipliers from $X$ to $Z$. For an extensive discussion on the problem of factorization we refer to [Sc10, KLM14, KLM19].

In the present paper we are not interested in the factorization problem itself (although we get factorization theorem for Hardy spaces as an outcome), but rather in a kind of its refinement (i.e. regularization as it is in the title) once we know that $Z=X \odot Y$ already holds. Namely, equality $Z=X \odot Y$ means that there exist constants $C, c>0$ such that, for a given $f \in Z$ there are $g \in X$ and $h \in Y$ satisfying $f=g h$ and

$$
c\|g\|_{X}\|h\|_{Y} \leqslant\|f\|_{Z} \leqslant C\|g\|_{X}\|h\|_{Y} .
$$

Suppose, however, that a function $f$ satisfies some regularity conditions (for example: it is holomorphic, smooth, non-increasing, or is a simple function). The question is

https://doi.org/10.5186/aasfm.2020.4545

2010 Mathematics Subject Classification: Primary 46E30, 46E15; Secondary 42B30, 46J15, 47 B35.

Key words: Symmetric spaces, Hardy spaces, Toeplitz operators, factorization. 
whether $g$ and $h$ may be chosen to be of the same class as $f$ and still satisfy the above estimates, possibly with other constants.

There is a plenty of classical theorems that may be seen as regularization of factorization. It seems that the most known is the one which says that each holomorphic function in the Hardy space $H^{1}$ (on the unit disc or half-plane) may be written as a product of two $H^{2}$ functions. It has a number of applications, for example in the recent proofs of Nehari theorem (see [Pe03] or [Le19]), or it is used in the proof of Hardy-Littlewood theorem on non-tangential maximal operator on $H^{1}$ (see [BS88, Ch. 5, Theorem 6.2]). More generally, factorization of $H^{p}$ functions have been used, among others, by Pisier in his proof of Jones interpolation theorem (see [Pi92]).

Regularization of factorization of non-increasing functions was already widely studied in [Ca64, KLM14, KLM19] and appeared to be one of the main tools thereinsee for example [KLM14, Lemma 7] or [KLM14, Theorem 1].

Motivated by those results, we decided to separate the problem of regularization of factorization from the mentioned context of applications and discuss its possible generalizations in different directions. We rather put our attention on general function spaces, while their underlying measure spaces are as simple as possible. In our paper we focus on three types of regularization - for step functions, for smooth functions and for holomorphic functions.

The paper is organized as follows. In the next section we collect definitions and preliminary considerations. In Section 3 we present a solution of the regularization problem for step functions, while in Section 4 we consider the same question for smooth functions. The main results of the paper are contained in Section 5, where we study regularization for holomorphic functions and characterize multipliers for abstract Hardy spaces on the disc. Then, in Section 6, we apply obtained results to the factorization of Toeplitz operators on Hardy spaces.

\section{Preliminaries}

Let $(\Omega, \Sigma, \mu)$ be a complete $\sigma$-finite and non-atomic measure space and let $L^{0}(\Omega)$ $:=L^{0}(\Omega, \Sigma, \mu)$ denotes the space of (equivalence classes of $\mu$-a.e. equal) real-valued measurable functions on $\Omega$ with the topology of convergence in measure on $\mu$-finite sets. The order $|f| \leqslant|g|$ means that $|f(\omega)| \leqslant|g(\omega)|$ for $\mu$-almost all $\omega \in \Omega$. A real quasi-Banach space $X:=X(\Omega) \subset L^{0}(\Omega)$ is called a quasi-Banach function lattice on $\Omega$ (quasi-Banach lattice or even q-BFL for brevity), whenever the following two conditions are satisfied:

(i) if $|f| \leqslant|g| \mu$-a.e. and $g \in X$, then $f \in X$ and $\|f\| \leqslant\|g\|$,

(ii) the characteristic function $\chi_{E}$ of any set $E \in \Sigma$ belongs to $X$ (equivalently, $\left.L^{\infty}(\Omega) \subset X\right)$.

The symbol $X_{+}$stands for the cone of positive elements of $X$. If $X$ is a Banach space we will write Banach lattice (or just BFL) instead of Banach function lattice. Through the paper we will consider two kinds of measure spaces. In Section 4 either $I=[0,1]$ or $I=[0, \infty)$ with the Lebesgue measure $m$, or the unit circle $\mathbf{T}:=$ $\left\{e^{i \theta}: \theta \in[0,2 \pi)\right\}$ with the normalized Lebesgue measure, also denoted by $m$, in the remaining sections.

Equality $X \equiv Y$ means that spaces are equal, as well as their quasi-norms. The symbol $\|\cdot\|_{X} \approx\|\cdot\|_{Y}$ will be used when the quasi-norms are equivalent.

A quasi-Banach lattice $X$ satisfies the Fatou property when given any sequence $\left(f_{n}\right) \subset X$ and $f \in L^{0}$ satisfying $0 \leqslant f_{n} \uparrow f \mu$-a.e. as $n \rightarrow \infty$ and $\sup _{n}\left\|f_{n}\right\|<\infty$, it 
follows that $f \in X$ and $\|f\|=\sup _{n}\left\|f_{n}\right\|$. A function $f \in X$ is said to be an order continuous element of $X$ if $0 \leq f_{n} \leqslant|f|$ with $f_{n} \rightarrow 0 \mu$-a.e. implies that $\left\|f_{n}\right\| \rightarrow 0$. The subspace of order continuous elements of $X$ is denoted by $X_{o}$. A space $X$ is said to be order continuous when $X=X_{o}$.

For $f \in L^{0}(\Omega)$, its distribution function is defined by $\mu_{f}(\lambda)=\mu(\{t \in \Omega:|f(t)|>$ $\lambda\}), \lambda \geqslant 0$. The non-increasing rearrangement $f^{*}$ of $f \in L^{0}$ is given by the formula

$$
f^{*}(t)=\inf \left\{\lambda>0: \mu_{f}(\lambda) \leqslant t\right\}, \quad t \geqslant 0 .
$$

A quasi-Banach lattice $X$ is called symmetric (or rearrangement invariant space) if for any $f \in X$ and $g \in L^{0}(\Omega)$ such that $\mu_{f}=\mu_{g}$ we have $g \in X$ and $\|f\|=\|g\|$. For $f \in L_{\text {loc }}^{1}$ one defines

$$
f^{* *}(t)=\frac{1}{t} \int_{0}^{t} f^{*}(s) d s .
$$

A symmetric Banach space $X \subset L_{\text {loc }}^{1}$ is called fully symmetric when for any $f \in X$ and $g \in L_{\text {loc }}^{1}(\Omega)$ such that $g^{* *}(t) \leqslant f^{* *}(t)$ for all $t>0$ we have $g \in X$ and $\|g\| \leqslant\|f\|$.

2.1. Multipliers and products. Let $X$ and $Y$ be a couple of quasi-Banach lattices. The space of multipliers $M(X, Y)$ is defined as

$$
M(X, Y)=\left\{f \in L^{0}: f g \in Y \text { for each } g \in X\right\}
$$

equipped with the quasi-norm

$$
\|f\|_{M(X, Y)}=\sup \left\{\|f g\|_{Y}:\|g\|_{X}=1\right\} .
$$

It particular, for $Y=L^{1}$, the space of pointwise multipliers is just the Köthe dual of $X$, i.e. $X^{\prime} \equiv M\left(X, L^{1}\right)$.

The notion of multipliers is intimately connected to the product of spaces. Recall that for two q-BFLs $X$ and $Y$ the product $X \odot Y$ is defined as

$$
X \odot Y=\{g h: g \in X, h \in Y\} .
$$

It's worth mentioning that, in general, even if $X, Y$ are Banach function lattices, then the product $X \odot Y$ doesn't need to be a Banach space. Indeed, the product space is, in general, a quasi-Banach space when equipped with the following quasi-norm

$$
\|f\|_{X \odot Y}=\inf \left\{\|g\|_{X}\|h\|_{Y}: f=g h, g \in X, h \in Y\right\} .
$$

We refer the reader to the recent papers [KLM14, Sc10] and references included therein.

\section{Regularization for factorization of step functions}

The following theorem may be viewed as a generalization of [KLR14, Lemma 3.1], since product spaces are closely connected with the Calderón-Lozanovskil construction (see [KLM14, Theorem 1(iv)]).

Theorem 3.1. Let $X$ and $Y$ be two fully symmetric spaces over $(I, m)$, where $I=[0,1]$ or $I=[0, \infty)$ and $m$ is the Lebesgue measure. Suppose that $f \in X \odot Y$ is a step function of the form

$$
f=\sum_{n=1}^{\infty} c_{n} \chi_{A_{n}}
$$


where $\left(c_{n}\right) \subset \mathbf{R} \backslash\{0\}$ and $\left(A_{n}\right) \subset I$ is any sequence of pairwise disjoint sets of finite measure. Then $\|f\|_{X \odot Y}$ is realized on elements of the same form, i.e.

$$
\|f\|_{X \odot Y}=\inf \left\{\|g\|_{X}\|h\|_{Y}:|f|=g h, g=\sum_{n=1}^{\infty} a_{n} \chi_{A_{n}} \in X_{+}, h=\sum_{n=1}^{\infty} b_{n} \chi_{A_{n}} \in Y_{+}\right\} .
$$

If both $X, Y$ satisfy the Fatou property, then the above formula holds with minimum instead of infimum.

Proof. Let $f=\sum_{n=1}^{\infty} c_{n} \chi_{A_{n}}, f \geqslant 0$, belongs to the product space $X \odot Y$. Choose any $g \in X_{+}, h \in Y_{+}$satisfying $f=g h$. Define the averaging operator $T$ by the formula

$$
T: g \mapsto \sum_{n=1}^{\infty} \frac{1}{m\left(A_{n}\right)}\left(\int_{A_{n}} g d m\right) \chi_{A_{n}}
$$

It is evident, that $T$ is a contraction on $L^{1}$ as well as on $L^{\infty}$. Thus, the CalderónMityagin interpolation theorem (cf. [KPS82, Theorem 4.3, p. 95]) implies that it is also a contraction on $X$ and on $Y$, since both spaces are fully symmetric. Define

$$
g^{\prime}=\sum_{n=1}^{\infty} \frac{1}{m\left(A_{n}\right)}\left(\int_{A_{n}} g d m\right) \chi_{A_{n}}, \quad h^{\prime}=\sum_{n=1}^{\infty} \frac{1}{m\left(A_{n}\right)}\left(\int_{A_{n}} h d m\right) \chi_{A_{n}} .
$$

Of course, $\left\|g^{\prime}\right\|_{X} \leqslant\|g\|_{X}$ and $\left\|h^{\prime}\right\|_{Y} \leqslant\|h\|_{Y}$. Moreover, using the inverse Chebyshev inequality [KLM14, Lemma 1], for a fixed $n$, we obtain

$$
c_{n}=\frac{1}{m\left(A_{n}\right)} \int_{A_{n}} g h d m \leqslant \frac{1}{m\left(A_{n}\right)} \int_{A_{n}} g d m \frac{1}{m\left(A_{n}\right)} \int_{A_{n}} h d m .
$$

Applying the above inequality to each set $A_{n}$ we obtain $f \leqslant g^{\prime} h^{\prime}$. Therefore,

$$
\begin{aligned}
\|f\|_{X \odot Y} & =\inf \left\{\|g\|_{X}\|h\|_{Y}: f=g h, g \in X_{+}, h \in Y_{+}\right\} \\
& \geqslant \inf \left\{\|g\|_{X}\|h\|_{Y}: f \leqslant g h, g=\sum_{n=1}^{\infty} a_{n} \chi_{A_{n}} \in X_{+}, h=\sum_{n=1}^{\infty} b_{n} \chi_{A_{n}} \in Y_{+}\right\} \\
& =\inf \left\{\|g\|_{X}\|h\|_{Y}: f=g h, g=\sum_{n=1}^{\infty} a_{n} \chi_{A_{n}} \in X_{+}, h=\sum_{n=1}^{\infty} b_{n} \chi_{A_{n}} \in Y_{+}\right\}
\end{aligned}
$$

since it follows by [KLM14, Proposition 1] that in definition of the quasi-norm on product space one can equivalently put $f \leqslant g h$ instead of $f=g h$.

To see that the above infimum is attained when both spaces have the Fatou property it is enough to use Proposition 1 from [Re88] together with Theorem 1(iv) from [KLM14] and apply the above averaging to elements that realize $\|f\|_{X \odot Y}$.

\section{Regularization for factorization of smooth functions}

We have seen in the previous section, that regularization is possible for step functions. It is of interest to ask if similar property holds for smooth functions. Let $f=g h$, where $g \in X, h \in Y$ with $\|f\|_{X \odot Y} \approx\|g\|_{X}\|h\|_{Y}$ and assume that $f$ is smooth (i.e., $f$ is in $C^{k}(\mathbf{T})$ for some $k \in \mathbf{N}$ or $k=\infty$ ). Can we choose $g, h$ to be smooth as well, keeping $\|f\|_{X \odot Y} \approx\|g\|_{X}\|h\|_{Y}$ ? The following theorem settles this question for symmetric spaces on $\mathbf{T}$.

Theorem 4.1. Let $X$ and $Y$ be two fully symmetric spaces on $\mathbf{T}$ with the normalized Lebesgue measure $m$. Suppose that $f \in X \odot Y$ and $f \in C^{k}$, where $k \in \mathbf{N}$ 
or $k=\infty$. Then for each $\varepsilon>0$ there are $g \in X, h \in Y$, both of the class $C^{k}$, such that $f=g h$ and

$$
(1-\varepsilon)\|g\|_{X}\|h\|_{Y} \leqslant\|f\|_{X \odot Y} \leqslant\|g\|_{X}\|h\|_{Y} .
$$

Proof. Notice that if one of spaces $X$ or $Y$ is equal to $L^{\infty}$, then the statement trivially holds, since $X \odot L^{\infty} \equiv X$ and the equality $f=f \chi_{\mathbf{T}}$ gives the required factorization. Thus, we may assume that both $X$ and $Y$ are different from $L^{\infty}$.

Suppose firstly, that $Y$ is order continuous. Let $f \in X \odot Y$ be of the class $C^{k}$ and fix $\varepsilon>0$. There exist $g \in X$ and $h \in Y$ such that $f=g h$ and

$$
(1-\varepsilon / 2)\|g\|_{X}\|h\|_{Y} \leqslant\|f\|_{X \odot Y} \leqslant\|g\|_{X}\|h\|_{Y} .
$$

Of course, we may assume that $g \geqslant 0$. Then, for an arbitrary $\delta>0$ we define

$$
A_{\delta}=\{t \in \mathbf{T}: g(t)<\delta\}, \quad A_{\delta}^{\prime}=\mathbf{T} \backslash A_{\delta},
$$

and we put

$$
u_{\delta}=g \chi_{A_{\delta}^{\prime}}+\delta \chi_{A_{\delta}}, \quad v_{\delta}=f / u_{\delta}
$$

Notice that since $L^{\infty} \subset X$ and $0<u_{\delta} \leqslant g+\delta \chi_{\mathbf{T}}$, we get $\left\|u_{\delta}\right\|_{X} \rightarrow\|g\|_{X}$ as $\delta \rightarrow 0^{+}$. Moreover,

$$
\left|v_{\delta}\right|=\frac{|f|}{u_{\delta}} \leqslant \frac{|f|}{g}=|h|
$$

In consequence, $\left\|v_{\delta}\right\|_{Y} \leqslant\|h\|_{Y}$ for each $\delta>0$.

Define further

$$
u_{\delta}^{n}(t)=u_{\delta} * K_{n}(t)=\frac{1}{2 \pi} \int_{0}^{2 \pi} u_{\delta}(s) K_{n}(t-s) d s, \quad t \in[0,2 \pi],
$$

where $K_{n}$ is the Fejér kernel

$$
K_{n}\left(e^{i \theta}\right)=\sum_{k=-n}^{n}\left(1-\frac{|k|}{n+1}\right) e^{i k \theta}=\frac{1}{n+1}\left(\frac{\sin \frac{(n+1) \theta}{2}}{\sin \frac{\theta}{2}}\right)^{2}, \quad \theta \in[0,2 \pi],
$$

(see, for example, [Ka76, p. 12]). Then, using Calderón-Mityagin interpolation theorem (cf. [KPS82, Theorem 4.3, p. 95]) and the fact that convolution with the Fejér kernel is a contraction on $L^{1}$ and on $L^{\infty}$, we get $\left\|u_{\delta}^{n}\right\|_{X} \leqslant\left\|u_{\delta}\right\|_{X}$. Moreover, from the fact that

$$
u_{\delta} * K_{n}(t)-\delta=u_{\delta} * K_{n}(t)-\delta \chi_{\mathbf{T}} * K_{n}(t)=\left[u_{\delta}-\delta \chi_{\mathbf{T}}\right] * K_{n}(t) \geqslant 0,
$$

where the first equality is due to the fact that $\left\|K_{n}\right\|_{1}=1$ and the second one comes from $K_{n} \geqslant 0, n \in \mathbf{N}$, it follows that $u_{\delta}^{n}(t) \geqslant \delta$ for each $t \in \mathbf{T}$. In consequence,

$$
v_{\delta}^{n}=f / u_{\delta}^{n}
$$

is well defined and belongs to $C^{k}$, while $u_{\delta}^{n} \in C^{\infty}$. It is enough to notice that $\left\|v_{\delta}^{n}\right\|_{Y} \rightarrow\left\|v_{\delta}\right\|_{Y} \leqslant\|h\|_{Y}$ as $n \rightarrow \infty$. Indeed, since $u_{\delta}^{n} \rightarrow u_{\delta}$ a.e. on $\mathbf{T}$, as $n \rightarrow \infty$, we conclude that also $v_{\delta}^{n} \rightarrow v_{\delta}$ a.e. on $\mathbf{T}$ when $n \rightarrow \infty$. Moreover, $\left|v_{\delta}^{n}\right| \leqslant|f| / \delta \leqslant C$ for some constant $C>0$. Therefore, by order continuity of $Y$ we have even more than needed, i.e. $\left\|v_{\delta}^{n}-v_{\delta}\right\|_{Y} \rightarrow 0$ as $n \rightarrow \infty$. In particular, $\left\|v_{\delta}^{n}\right\|_{Y} \rightarrow\left\|v_{\delta}\right\|_{Y}$ as $n \rightarrow \infty$. Finally, we can see that selecting $\delta>0$ small and $n$ large enough we get

$$
(1-\epsilon)\left\|u_{\delta}^{n}\right\|_{X}\left\|v_{\delta}^{n}\right\|_{Y} \leqslant\|f\|_{X \odot Y} \leqslant\left\|u_{\delta}^{n}\right\|_{X}\left\|v_{\delta}^{n}\right\|_{Y}
$$

with $f=u_{\delta}^{n} v_{\delta}^{n}$, as required. The second inequality in (4.1) is always true by the definition of $X \odot Y$.

If $X$ is order continuous, then the proof is analogous. Suppose now that both $X, Y$ are not order continuous, but as we noticed at the beginning, both are different 
than $L^{\infty}$. Then $X \odot Y_{o}$ is order continuous (see [KLM14, Corollary 1], cf. [Re88, Proposition 4]). Further, also $X \odot Y \neq L^{\infty}$, which means that $L^{\infty} \subset(X \odot Y)_{o}$. In consequence, $(X \odot Y)_{o} \equiv X \odot Y_{o}$, as order continuous subspaces of the same space $X \odot Y$, while equality of norms follows from Theorem 3.1, since simple functions are dense in both $X \odot Y_{o}$ and $(X \odot Y)_{o}$. From the fact that $f \in C^{k}$ it follows that $f \in L^{\infty}$ and $L^{\infty} \subset(X \odot Y)_{o} \equiv X \odot Y_{o}$. Thus we can apply the previous part to $X \odot Y_{o}$, because $Y_{o}$ is always fully symmetric for any symmetric space $Y$ (see [KPS82, Theorem 4.10, p. 105]).

Notice that the analogous problem to the one considered in Theorem 4.1, has been already studied for Sobolev spaces. To be more specific, one may ask if a function in Sobolev space (or another space of smooth functions) may be factorized by functions from another Sobolev spaces. For example, Miyachi in [Mi95] showed that it is not the case for De Vore-Sharpley spaces $C_{p}^{\alpha, k}$ (see [Mi95] for definitions), i.e., each function from $C_{p}^{\alpha, k}$ admits weak factorization subject to $C_{p_{1}}^{\alpha, k}$ and $C_{p_{2}}^{\alpha, k}$ spaces, but not necessarily the strong one (for origins of weak factorization see [CRW76]). One of the reason for such a phenomenon is that factorizing by spaces with prescribed smoothness one needs to control at the same time the size of the function itself, as well as its derivatives, while relation $f=g h$ allows to control easily only factorizing functions itself, but not their derivatives. Of course, in Theorem 4.1 we say nothing about the size of derivatives of factors, except those derivatives exist.

\section{Multipliers and products for Hardy spaces - regularization for holomorphic functions}

In this section we will consider quasi-Banach function lattices over $\mathbf{T}=\left\{e^{i \theta}: \theta \in\right.$ $[0,2 \pi]\}$. The term complex function lattice refers to the complexification of a real function lattice $X$, i.e., if $X$ denotes the (real) space, the complexification $X(\mathbf{C})$ of $X$ is the space of all complex-valued measurable functions $f$ on $\mathbf{T}$ such that the element $|f|$ defined by $|f|(\mathbf{T})=|f(\mathbf{T})|$ for $t \in \mathbf{T}$ is in $X$ and $\|f\|=\||f|\|_{X}$. For simplicity of presentation, we will rather write about function lattices instead of complex function lattices and avoid the use of the symbol $X(\mathbf{C})$, remembering however, that in this and the next section all spaces are complex.

Moreover, we will consider Hardy spaces defined by quasi-Banach spaces. However, to avoid some pathological situations we shall restrict slightly the class of all quasi-Banach lattices. In particular, we will need upper semi-continuity of respective quasi-norm, that is $\left\|f_{n}\right\| \rightarrow 0$ and $f_{n} \geqslant 0$ implies $\left\|f+f_{n}\right\| \rightarrow\|f\|$. In order to ensure it, we will assume that for a quasi-Banach lattice $X$ there exists $p \geqslant 1$ such that $X^{(p)}$ is the Banach space, where $X^{(p)}$ is the $p$-convexification (resp., $p$-concavification, when $p \in(0,1))$ of $X$ defined by

$$
X^{(p)}=\left\{f \in L^{0}:|f|^{p} \in X\right\}
$$

and equipped with the quasi-norm $\|f\|_{X^{(p)}}=\left\||f|^{p}\right\|_{X}^{1 / p}$. To see that such assumption ensures continuity of the quasi-norm $\|\cdot\|_{X}$, let $\left\|f_{n}\right\|_{X} \rightarrow 0, f \in X$ and assume that $p$-convexification $X^{(p)}$ is a Banach space. Then

$$
\begin{aligned}
\left\|f+f_{n}\right\|_{X} & =\left\|f+f_{n}\right\|_{\left(X^{(p)}\right)^{(1 / p)}}=\left\|\left|f+f_{n}\right|^{1 / p}\right\|_{X^{(p)}}^{p} \\
& \leqslant\left\||f|^{1 / p}+\left|f_{n}\right|^{1 / p}\right\|_{X^{(p)}}^{p} \leqslant\left(\left\||f|^{1 / p}\right\|_{X^{(p)}}+\left\|\left|f_{n}\right|^{1 / p}\right\|_{X^{(p)}}\right)^{p} \\
& \rightarrow\left\||f|^{1 / p}\right\|_{X^{(p)}}^{p}=\|f\|_{X} .
\end{aligned}
$$


Notice finally that such the assumption is slightly stronger than the so called $L$ convexity, which says just that $X$ is $p$-convex for some $p>0$ or, equivalently, $X^{(p)}$ is normable for some $p>0$ (see [KR09, Ka84] for thorough discussion of this notion).

To shorten the notion we define the following class of function lattices.

Definition 5.1. A quasi-Banach function lattice $X$ on $\mathbf{T}$ belongs to the class $Q$ if there exists $p \geqslant 1$ such that $X^{(p)}$ is a Banach space and $L^{\infty} \subset X \subset L^{1 / p}$.

The latter inclusions will be useful in a moment when we consider inner-outer factorization in Hardy spaces built upon $X \in Q$. Notice that when $X$ is a symmetric space and $X^{(p)}$ is a Banach space, then $X^{(p)}$ is a symmetric space and thus $X^{(p)} \subset$ $L^{1}(\mathbf{T})$ or, equivalently, $X \subset L^{1 / p}(\mathbf{T})$. Of course, each symmetric space contains $L^{\infty}$ as well.

Finally, we point out that the class $Q$ is closed with respect to pointwise multipliers and products. That is, if $X \subset Y$ and $X, Y \in Q$, then also $M(X, Y) \in Q$. In fact, if $X^{(p)}$ and $Y^{(q)}$ are Banach spaces for some $p, q \geqslant 1$, then $X^{(r)}$ and $M(X, Y)^{(r)}$ are Banach spaces with $r=\max \{p, q\}$ since $M(X, Y)^{(r)} \equiv M\left(X^{(r)}, Y^{(r)}\right)$. Evidently, $L^{\infty} \subset M(X, Y) \subset Y$. Similarly, if $X, Y \in Q$, then also $X \odot Y \in Q$.

Let $H(\mathbf{D})$ be the space of all analytic functions on $\mathbf{D}=\{z \in \mathbf{C}:|z|<1\}$. Denote by $H^{+}(\mathbf{D})$ the set of those functions $f \in H(\mathbf{D})$ such that the radial limit $f_{*}$ exists almost everywhere on $\mathbf{T}$, where

$$
f_{*}(t):=\lim _{r \rightarrow 1^{-}} f_{r}(t), \quad t \in \mathbf{T},
$$

and $f_{r}(t):=f(r t)$ for $t \in \mathbf{T}$ and $r \in[0,1)$.

Let $X \in Q$. We define the Hardy space $H[X]$ as

$$
H[X]:=\left\{f \in H^{+}: f_{*} \in X\right\}
$$

with the quasi-norm

$$
\|f\|_{H[X]}=\left\|f_{*}\right\|_{X} .
$$

Then $H[X]$ is a quasi-Banach space and-by identifying $f \in H[X]$ with $f_{*}$-may be seen as a closed subspace of $X$. The detailed study of spaces $H[X]$ has been presented in [BS77, MM10, KS19]. Let us also note that for particular spaces $X$, the above method produces variants of Hardy spaces widely studied in the literature. For example, if $X=L^{p}, p \in(0, \infty)$, then $H[X]=H^{p}$ is the standard Hardy space. If $X=L^{\varphi}$ is an Orlicz space, then $H^{\varphi}=H\left[L^{\varphi}\right]$ is the Hardy-Orlicz space (see [Le71]). Let us highlight as well that there is another, eventually equivalent, definition of Hardy spaces $H[X]$ via the growth of the function $r \mapsto\left\|f_{r}\right\|_{X}$ (see [MM10] for more details).

Let $X, Y$ be in $Q$. We wish to consider pointwise multipliers between $H[X]$ and $H[Y]$, which may be defined either as

$$
M(H[X], H[Y])=\{f \in H(\mathbf{D}): f g \in H[Y] \text { for each } g \in H[X]\},
$$

or as

$$
M(H[X], H[Y])=\left\{f \in H^{+}: f_{*} g_{*} \in Y \text { for each } g \in H[X]\right\} .
$$

Notice, however, that both definitions describe the same object. This follows from the fact that if $f, g \in H^{+}(\mathbf{D})$, then $(f g)_{*}=f_{*} g_{*}$ and vice versa, i.e., if $(f g)_{*}$ and $g_{*}$ exist, then also $f_{*}$ exists, since $g_{*}(t) \neq 0$ for almost all $t \in \mathbf{T}$ for a non-zero function $g$. Moreover, in both cases $M(H[X], H[Y])$ is a subspace of $H[Y]$ since 
constant functions belong to $H[X]$. We consider the space $M(H[X], H[Y])$ with the associated quasi-norm

$$
\|f\|_{M(H[X], H[Y])}=\sup \left\{\|f g\|_{H[Y]}:\|g\|_{H[X]}=1\right\} .
$$

Further, we will accept a slight discrepancy in notation and we will identify function on the disc with its radial limit. This will allow us to consider the following pointwise multipliers from a space of analytic functions to a space of measurable functions

$$
M(H[X], Y)=\left\{f \in L^{0}: f g_{*} \in Y \text { for each } g \in H[X]\right\}
$$

equipped with an associated functional

$$
\|f\|_{M(H[X], Y)}=\sup \left\{\left\|f g_{*}\right\|_{Y}:\|g\|_{H[X]} \leqslant 1\right\} .
$$

We start with the following result.

Theorem 5.2. Let $X, Y \in Q$ be such that $X \subset Y$. Then

(i) $H[M(X, Y)] \equiv M(H[X], H[Y])$,

(ii) $M(X, Y) \equiv M(H[X], Y)$.

Proof. (i) Suppose that $f \in H[M(X, Y)]$. Then for each $h \in X, f_{*} h \in Y$. In particular, $f_{*} g_{*} \in Y$ for each $g \in H[X]$. Since $f g \in H(\mathbf{D})$ and $(f g)_{*}=f_{*} g_{*}$, it follows that $f \in M(H[X], H[Y])$ and

$$
\begin{aligned}
\|f\|_{H[M(X, Y)]} & =\sup \left\{\left\|f_{*} h\right\|_{Y}:\|h\|_{X} \leqslant 1\right\} \geqslant \sup \left\{\left\|f_{*} g_{*}\right\|_{Y}:\|g\|_{H[X]} \leqslant 1\right\} \\
& =\|f\|_{M(H[X], H[Y])} .
\end{aligned}
$$

Let now $f \in H[Y]$ and assume that $f \notin H[M(X, Y)]$. Thus there exists $h \in X$ such that $h f_{*} \notin Y$. Take $\delta>0$ and define

$$
v_{\delta}=|h|+\delta \chi_{\mathbf{T}} .
$$

It is clear that $v_{\delta} f_{*} \notin Y$. However, since $v_{\delta} \in X$ and $v_{\delta}>\delta$, then $\log \left|v_{\delta}\right| \in L^{1}$. We put $g_{\delta}$ to be an outer function of $v_{\delta}$ (see [Du70, p. 24]), i.e.,

$$
g_{\delta}(z):=\exp \left(\frac{1}{2 \pi} \int_{-\pi}^{\pi} \frac{e^{i \theta}+z}{e^{i \theta}-z} \log \left|v_{\delta}\right|(\theta) d \theta\right) .
$$

It is known that $\left(g_{\delta}\right)_{*}=v_{\delta}$ and hence $\left(g_{\delta}\right)_{*} f_{*} \notin Y$ as well. This means that $f \notin$ $M(H[X], H[Y])$, since $g_{\delta} \in H[X]$. Therefore,

$$
H[M(X, Y)]=M(H[X], H[Y])
$$

as sets.

To prove the required inequality on quasi-norms let $\varepsilon>0$ and choose $h \in X$ such that $\|h\|_{X}<1$ and

$$
\|f\|_{H[M(X, Y)]} \leqslant\left\|f_{*} h\right\|_{Y}+\varepsilon .
$$

Applying constructions (5.1) and (5.2) to such chosen $h$ we get that for each $\delta>0$ the outer function $g_{\delta}$ satisfies the equality $\left|\left(g_{\delta}\right)_{*}\right|=\left|v_{\delta}\right|=|h|+\delta \chi_{\mathbf{T}}$. Thanks to the upper semi-continuity of quasi-norm of $X$ we get $\left\|\left(g_{\delta}\right)_{*}\right\|\left\|_{X}=\right\| v_{\delta}\left\|_{X} \rightarrow\right\| h \|_{X}$ as $\delta \rightarrow 0$. Since $|h| \leqslant\left|\left(g_{\delta}\right)_{*}\right|$ we conclude

$$
\|f\|_{H[M(X, Y)]}-\varepsilon \leqslant\left\|f_{*} h\right\|_{Y} \leqslant\left\|f_{*}\left(g_{\delta}\right)_{*}\right\|_{Y} \leqslant\|f\|_{M(H[X], H[Y])},
$$

where the last inequality holds for $\delta>0$ so small that $\left\|\left(g_{\delta}\right)_{*}\right\|_{X}<1$. This means that $\|f\|_{H[M(X, Y)]} \leqslant\|f\|_{M(H[X], H[Y])}$ and the proof of (i) is finished.

The proof of (ii) is analogous and therefore will be omitted. 
In order to study products of Hardy spaces we will need the following auxiliary result.

Proposition 5.3. [Ka04, Proposition 5.1] If $\log _{+}|f|, \log _{+}|g| \in L^{1}(\mathbf{T})$ and $f g \in$ $H^{1} \backslash\{0\}$, then $\log |f|, \log |g| \in L^{1}$.

Point (i) of the theorem below is a generalization of [Ka04, Theorem 5.2] and [Le19, Theorem 3.6]. It fits into subject of the paper, since it should be treated as a holomorphic regularization for factorization. The second point will be applied in the next section.

Theorem 5.4. Let $X, Y \in Q$. Then

(i) $H[X] \odot H[Y] \equiv H[X \odot Y]$,

(ii) $H[X] \odot Y \equiv X \odot Y$.

Proof. (i) The inclusion $H[X] \odot H[Y] \subset H[X \odot Y]$ with $\|f\|_{H[X \odot Y]} \leqslant\|f\|_{H[X] \odot H[Y]}$ is evident. We need to show that $H[X \odot Y] \subset H[X] \odot H[Y]$ with $\|f\|_{H[X \odot Y]} \geqslant$ $\|f\|_{H[X] \odot H[Y]}$. Let $f \in H(X \odot Y)$. Since for some $p_{0}, p_{1}>0, X \subset L^{p_{0}}, Y \subset L^{p_{1}}$, we have $X \odot Y \subset L^{r}$, where $1 / r=1 / p_{0}+1 / p_{1}$. In consequence, $f \in H^{r}$ and so $f$ admits the inner-outer factorization (see [Du70, Theorem 2.8]), i.e., $f=\phi F$, where $\phi$ is an inner and $F$ is an outer function, and $\left|f_{*}\right|=\left|F_{*}\right|$. Let $g \in X, h \in Y$ be such that $F_{*}=g h$. We have $F^{1 / r} \in H^{1}$ and $\left(F_{*}\right)^{1 / r}=\psi|g|^{1 / r}|h|^{1 / r}$, where $\psi=F_{*} /\left|F_{*}\right|$. Evidently, $\log _{+}|g|^{1 / r}, \log _{+}|h|^{1 / r} \in L^{1}$ and from Proposition 5.3 we get that $\log |g|^{1 / r}, \log |h|^{1 / r} \in L^{1}$, which means that also $\log |g|, \log |h| \in L^{1}$. It is therefore enough to take

$$
G(z)=\exp \left(\frac{1}{2 \pi} \int_{-\pi}^{\pi} \frac{e^{i \theta}+z}{e^{i \theta}-z} \log |g(\theta)| d \theta\right) .
$$

Then $G$ is an outer function of $g$ and $\left|G_{*}\right|=|g|$. Consequently, taking $v=F / G$ we see that $v$ is analytic on $\mathbf{D}$ and

$$
\left|v_{*}\right|=\frac{\left|F_{*}\right|}{|g|}=|h| .
$$

Therefore, $F=G v$ and so

$$
f=\phi G v,
$$

while $\|\phi G\|_{H[X]}=\|g\|_{X}$ and $\|v\|_{H[Y]}=\|h\|_{Y}$, which gives the required factorization.

(ii) Once again, the inclusion $H[X] \odot Y \subset X \odot Y$ and inequality $\|f\|_{X \odot Y} \leqslant$ $\|f\|_{H[X] \odot Y}$ are evident. We will prove the opposite. Let $h \in X \odot Y$ and choose $f \in X, g \in Y$ such that $h=f g$ and $\|h\|_{X \odot Y} \geqslant\|f\|_{X}\|g\|_{Y}-\varepsilon$. We may assume that $0 \leqslant f$ and $\|f\|_{X}=1$. For $\delta>0$ define

$$
A_{\delta}=\{t \in \mathbf{T}: f(t)<\delta\}, \quad A_{\delta}^{\prime}=\mathbf{T} \backslash A_{\delta},
$$

and put

$$
u_{\delta}=f \chi_{A_{\delta}^{\prime}}+\delta \chi_{A_{\delta}} .
$$

Then $1=\|f\|_{X} \leqslant\left\|u_{\delta}\right\|_{X}$. We choose also

$$
v_{\delta}=g \chi_{A_{\delta}^{\prime}}+\frac{h}{\delta} \chi_{A_{\delta}} .
$$

Thus $\left|v_{\delta}\right| \leqslant|g|$ and we have

$$
h=u_{\delta} v_{\delta}
$$


Similarly, as in (5.2) from the proof of Theorem 5.2 we may choose $a_{\delta}$ to be the outer function of $u_{\delta}$, since $\log u_{\delta} \in L^{1}$. Then $\left|\left(a_{\delta}\right)_{*}\right|=u_{\delta}$. Taking

$$
b_{\delta}=\frac{v_{\delta} u_{\delta}}{\left(a_{\delta}\right)_{*}}
$$

we obtain $\left|b_{\delta}\right|=\left|v_{\delta}\right|$ and $h=\left(a_{\delta}\right)_{*} b_{\delta}$, while $a_{\delta} \in H[X]$. Moreover,

$$
\|h\|_{H[X] \odot Y} \leqslant\left\|a_{\delta}\right\|_{H[X]}\left\|b_{\delta}\right\|_{Y} \leqslant\left\|a_{\delta}\right\|_{H[X]}\|g\|_{Y} \rightarrow\|f\|_{X}\|g\|_{Y} \leqslant\|h\|_{X \odot Y}+\varepsilon,
$$

when $\delta \rightarrow 0$. In consequence, we get

$$
\|h\|_{H[X] \odot Y} \leqslant\|h\|_{X \odot Y}
$$

as required.

As a corollary from the above theorems we get immediately the following Lozanovskiı's like factorization theorem for Hardy type spaces.

Corollary 5.5. Let $X, Y \in Q$ and assume that $X$ factorizes $Y$, i.e.,

$$
X \odot M(X, Y) \equiv Y \text {. }
$$

Then $H[X]$ factorizes $H[Y]$, i.e.,

$$
H[X] \odot M(H[X], H[Y]) \equiv H[Y] .
$$

Proof. Using Theorems 5.2 and 5.4 and our assumption we have

$$
H[X] \odot M(H[X], H[Y]) \equiv H[X] \odot H[M(X, Y)] \equiv H[X \odot M(X, Y)] \equiv H[Y]
$$

\section{Application to factorization of Toeplitz operators}

In this section we apply the previously obtained results to study of factorization of Toeplitz operators on Hardy spaces. Consider the Riesz projection $P$ which is formally defined on $L^{1}$ by

$$
P: f \mapsto \sum_{k=0}^{\infty} \widehat{f}(k) e^{i t},
$$

where $\widehat{f}(k)$ stands for the $k$-th Fourier coefficient of $f$. It is known, that $P$ is bounded on a symmetric Banach function space $X$ if and only if $X$ has nontrivial Boyd indices, i.e. $p_{X}, q_{X} \in(1, \infty)$ (see [FGJ73], while definition of Boyd indices and their properties may be found in [LT79, p. 131]).

Assume also that $X, Y$ are Banach function spaces such that $L^{\infty} \subset X \subset Y \subset L^{1}$ and the Riesz projection $P$ is bounded on $Y$. Then for each $a \in M(X, Y)$ the Toeplitz operator $T_{a}: H[X] \rightarrow H[Y]$ defined as

$$
T_{a}: f \mapsto P(a f)
$$

is bounded with a norm $\left\|T_{a}\right\| \leqslant\|P\|_{Y \rightarrow Y}\|a\|_{M(X, Y)}$ (see [To87, JPS84, Le19, KS19] for studies of Toeplitz operators acting between distinct Hardy spaces).

Below we present how results from the previous section may be used to factorize Toeplitz operators acting between Hardy spaces. It may be seen as a version of the classical Maurey factorization theorem for Toeplitz operators. Notice, however, that Hardy spaces are not Banach lattices, thus one cannot apply Maurey's theorem directly (see also [AK07] for discussion in the subject). 
Theorem 6.1. Let $X, Y$ be Banach function lattices with the Fatou property such that $L^{\infty} \subset X \subset Y \subset L^{1}$ and the Riesz projection $P$ is bounded on $Y$. If $X$ is $p$-convex and $Y$ is $p$-concave for some $p \in(1, \infty)$, then each Toeplitz operator $T_{a}: H[X] \rightarrow H[Y]$, where $a \in M(X, Y)$, factorizes strongly through $H^{p}$, i.e., there exist $b \in M\left(L^{p}, Y\right)$ and $\phi \in H\left[M\left(X, L^{p}\right)\right]$ such that $a=b \phi$ and the following diagram commutes

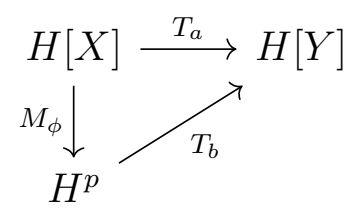

where $M_{\phi}: f \mapsto \phi f$ is the multiplication operator with a symbol $\phi$.

Proof. Our assumption together with the outcome of [Sc10, Theorems 3.3, 3.4 and 3.8] implies that

$$
X \odot M(X, Y)=Y, \quad L^{p} \odot M\left(L^{p}, Y\right)=Y, \quad X \odot M\left(X, L^{p}\right)=L^{p} .
$$

In consequence,

$$
X \odot M\left(X, L^{p}\right) \odot M\left(L^{p}, Y\right)=L^{p} \odot M\left(L^{p}, Y\right)=Y=X \odot M(X, Y)
$$

and, applying Lemma 4 (i) from [KLM19], we get

$$
M\left(X, L^{p}\right) \odot M\left(L^{p}, Y\right)=M(X, Y) .
$$

Now, if $a \in M(X, Y)$ is a symbol of the Toeplitz operator $T_{a}: H[X] \rightarrow H[Y]$, then Theorem 5.4 (ii) gives a factorization

$$
a=\phi b,
$$

where $\phi \in H\left[M\left(X, L^{p}\right)\right]$ and $b \in M\left(L^{p}, Y\right)$. On the other hand, $H\left[M\left(X, L^{p}\right)\right]=$ $M\left(H[X], H^{p}\right)$ and so both operators

$$
M_{\phi}: H[X] \rightarrow H^{p} \quad \text { and } \quad T_{b}: H^{p} \rightarrow H[Y]
$$

are bounded. Thus, for $f \in H[X]$

$$
T_{a}(f)=P(a f)=P(b \phi f)=T_{b}\left(M_{\phi} f\right),
$$

which finishes the proof.

Remark 6.2. Let $X, Y, Z$ be Banach function lattices with the Fatou property such that $L^{\infty} \subset X \subset Z \subset Y \subset L^{1}$ and the Riesz projection $P$ is bounded on $Y$. One may then repeat the above proof to get factorization through $H[Z]$ space provided we know that

$$
M(X, Y)=M(X, Z) \odot M(Z, Y) .
$$

In fact, we assumed concavity and convexity of respective spaces in the above theorem only to get (6.1) for $Z=L^{p}$. For example, for a triple of Lorentz spaces it may be easily deduced from [KLM19] when (6.1) holds.

Acknowledgements. The first and the third author were supported by the National Science Center (Narodowe Centrum Nauki), Poland (project no. 2017/26/D/ $\mathrm{ST} 1 / 00060)$. 


\section{References}

[AS07] Anisimov, D. S., and S. V. Kislyakov: Strong factorization of operators defined on subspaces of analytic functions. - J. Math. Sci. (N.Y.) 141:5, 2007, 1511-1516.

[BS88] Bennett, C., and R. Sharpley: Interpolation of operators. - Academic Press, Boston, 1988.

[BS77] Bryskin, I. B., and A. A. Sedaev: Geometric properties of the unit ball in Hardy-type spaces. - J. Soviet Math. 8:1, 1977, 1-9.

[Ca64] Calderón, A. P.: Intermediate spaces and interpolation, the complex method. - Studia Math. 24, 1964, 113-190.

[CRW76] Colfman, R. R., R. Rochberg, and G. G. Weiss: Factorization theorems for Hardy spaces in several variables. - Ann. of Math. 103:3, 1976, 611-635.

[Du70] Duren, P.: Theory of $H^{p}$ spaces. - Academic Press, Boston, 1970.

[FGJ73] Fehér, F., D. GaşPar, and H. Johnen: Normkonvergenz von Fourierreihen in rearrangement invarianten Banachräumen. - J. Funct. Anal. 13, 1973, 417-434.

[JPS84] Janson, S., J. Peetre, and S. Semmes: On the action of Hankel and Toeplitz operators on some function spaces. - Duke Math. J. 51:4, 1984, 937-958.

[Ka84] Kalton, N. J.: Convexity conditions on non-locally convex lattices. - Glasgow Math. J. $25: 2,1984,141-152$.

[KR09] Kamińska, A., and Y. RaYnaud: Isomorphic copies in the lattice $E$ and its symmetrization $E^{(*)}$ with applications to Orlicz-Lorentz spaces. - J. Funct. Anal. 257:1, 2009, 271-331.

[KLR14] Kamińska, A., K. Leśnik, and Y. RaYnaud: Dual spaces to Orlicz-Lorentz spaces. Studia Math. 222:3, 2014, 229-261.

[Ka04] Karlovich, A. Yu.: Norms of Toeplitz and Hankel operators on Hardy type subspaces of rearrangement-invariant spaces. - Integral Equations Operator Theory 49:1, 2004, 43-64.

[KS19] Karlovich, A. Yu., and E. Shargorodsky: The Brown-Halmos theorem for a pair of abstract Hardy spaces. - J. Math. Anal. Appl. 472:1, 2019, 246-265.

[Ka76] Katznelson, Y.: An introduction to harmonic analysis. - Dover Publications, Inc., New York, 1976.

[KLM14] Kolwicz, P., K. Leśnik, and L. Maligranda: Pointwise products of some Banach function spaces and factorization. - J. Funct. Anal. 266:2, 2014, 616-659.

[KLM19] Kolwicz, P., K. Leśnik, and L. Maligranda: Symmetrization, factorization and arithmetic of quasi-Banach function spaces. - J. Math. Anal. Appl. 470:2, 2019, 1136-1166.

[KPS82] Krein, S. G., Yu. I. Petunin, and E. M. Semenov: Interpolation of linear operators. - Amer. Math. Soc., Providence, 1982.

[Le71] Leśniewicz, R.: On Hardy-Orlicz spaces I. - Comment. Math. 15, 1971, 3-56.

[Le19] LEŚNIK, K.: Toeplitz and Hankel operators between distinct Hardy spaces. - Studia Math. 249:2, 2019, 163-192.

[LT79] Lindenstrauss, J., and L. Tzafriri: Classical Banach spaces II. - Springer-Verlag, Berlin, 1979.

[Lo69] LozanovskIĬ, G. YA.: On some Banach lattices. - Sibirsk. Mat. Zh. 10:3, 1969, 584-599; English transl. in Siberian. Math. J. 10, 1969, 419-431.

[Ma89] Maligranda, L.: Orlicz spaces and interpolation. - Seminars in Math. 5, University of Campinas, Campinas, 1989.

[MM10] MastyŁo, M., and P. Mleczko: Solid hulls of quasi-Banach spaces of analytic functions and interpolation. - Nonlinear Anal. 73:1, 2010, 84-98. 
[Mi95] Mryachi, A.: Multiplication and factorization of functions in Sobolev spaces and in $C_{p}^{\alpha}$ spaces on general domains. - Math. Nachr. 176, 1995, 209-242.

[Pe03] Peller, V. V.: Hankel operators and their applications. - Springer-Verlag, New York, 2003.

[Pi92] Pisier, G.: Interpolation between $H^{p}$ spaces and noncommutative generalizations I. Pacific J. Math. 155:2, 1992, 341-368.

[Re88] ReISNER, S.: On two theorems of Lozanovskiū concerning intermediate Banach lattices. - Lecture Notes in Math. 1317, Springer-Verlag, Berlin, 1988, 67-83.

[Sc10] Schep, A. R.: Products and factors of Banach function spaces. - Positivity 14:2, 2010, 301-319.

[To87] Tolokonnikov, V. A.: Hankel and Toeplitz operators in Hardy spaces. - J. Soviet Math. 37, 1987, 1359-1364.

Received 13 March 2019 • Accepted 10 September 2019 